\section{Eficácia da associação de cipermetrina, clorpirifós, butóxido de piperonila e fluazuron contra larvas de Dermatobia hominis em bovinos naturalmente infestados}

\author{
Efficacy of the association of cypermethrin, chlorpyriphos, \\ piperonyl butoxide and fluazuron against larvae of \\ Dermatobia hominis in naturally infested cattle
}

\begin{abstract}
Diefrey Ribeiro Campos', Barbara Rauta de Avelar', Gabriela Ferreira de Oliveira', Mariana Silva Revoredo Alves², Debora Azevedo Borges ${ }^{2}$, Monique Taveira Medeiros ${ }^{2}$, Katherina Comendouros ${ }^{3}$ \& Fabio Barbour Scott ${ }^{3 *}$

'Médicos veterinários, MScs. Programa de Pós-graduação em Ciências Veterinárias, Universidade Federal Rural do Rio de Janeiro - UFRRJ, Seropédica, RJ, Brasil

2Médica-veterinária. Programa de Pós-graduação em Ciências Veterinárias, Universidade Federal Rural do Rio de Janeiro UFRRJ, Seropédica, RJ, Brasil

${ }^{3}$ Médico veterinário, DSc, Professor. Departamento de Parasitologia Animal, Instituto de Veterinária, Universidade Federal Rural do Rio de Janeiro - UFRRJ, Seropédica, RJ, Brasil
\end{abstract}

\section{Resumo}

O objetivo deste trabalho foi avaliar a eficácia de uma formulação pur-on contendo a associação de cipermetrina (5\%), clorpirifós (7\%), butóxido de pireronila (5\%) e fluazuron (2,5\%) no controle da mosca D. hominis em bovinos naturalmente infestados. Foram utilizados 20 bovinos, mestiços, infestados naturalmente por $D$. hominis. Os animais foram divididos em dois grupos, controle (não tratado) e medicado, contendo 10 bovinos em cada, com base nas contagens de larvas vivas antes do tratamento. Os animais do grupo tratado foram medicados com a associação de cipermetrina ( $5 \mathrm{mg} / \mathrm{kg}$ ), clorpirifós (7mg/kg), butóxido de pireronila ( $5 \mathrm{mg} / \mathrm{kg}$ ) e fluazuron $(2,5 \mathrm{mg} / \mathrm{kg})$, na dose de $1 \mathrm{ml}$ para cada 10 quilos de peso vivo, pela via tópica ("pour on") em dose única. O número médio de larvas vivas no grupo controle foi $24,4 \pm 8,11,23,2 \pm 6,49,25,9 \pm 8,97$ e no grupo tratado $24,7 \pm 8,34,1,1 \pm 1,04$ e 0,7 $\pm 0,78$ nos dias zero, sete e 14 dias após o tratamento. A eficácia bernicida foi avaliada sete e 14 dias após o tratamento, a partir da contagem dos nódulos contendo larvas vivas nos animais dos dois. A eficácia da associação testada neste estudo foi de 95,26\% e 97,30\% nos dias sete e 14 respectivamente. A associação de cipermetrina, clorpirifós, butóxido de pireronila e fluazuron na formulação "pour on" é eficaz no controle de $D$. hominis em bovinos naturalmente infestados.

Palavras-chaves: controle, berne, piretróide, organosfosforado.

\begin{abstract}
The objective of this study was to evaluate the efficacy of a product ectoparasiticide containing an association of cypermethrin, chlorpyrifos, butoxide pireronila and fluazuron in formulating "pour on" the fly control $D$. hominis in cattle naturally infested. For this, were used twenty bovines, half-breed, without distinction of sex or age. All animals included in the study had naturally occurring $D$. hominis larvae on both sides of the body. The animals in the treated group were medicated with the combination of cypermethrin (5mg / kg), chlorpyrifos (7mg / kg), pireronyl butoxide (5mg / kg) and fluazuron $(2.5 \mathrm{mg} / \mathrm{kg}$ ) at a dose of $1 \mathrm{ml}$ for every $10 \mathrm{~kg}$ live weight, "pour on" in single-dose. The larvicidal efficacy for $D$. hominis efficacy was evaluated seven and 14 days after the treatment, from the nodule count of live larvae in the animals of the two groups. The mean number of live larvae in the control group was $24.4 \pm 8.11,23.2 \pm 6.49,25.9 \pm 8.97$ and in the treated group $24.7 \pm 8.34,1.1 \pm 1,04$ and $0.7 \pm 0.78$ on days zero, seven and 14 days after treatment The efficacy of the association tested in this study was $95.26 \%$ and $97.30 \%$ on days 7 and 14 respectively. The association of cypermethrin, chlorpyrifos, pyreronyl butoxide and fluazuron in the pour-on formulation is effective in controlling D. hominis in naturally infested cattle.
\end{abstract}

Keywords: control, warble fly, pyrethroid, organophosphorus.

\section{BJ $\mathrm{M}$ \\ Brazilian Journal of Veterinary Medicine}

p-ISSN 0100-2430

Como citar: Campos, D. R., De Avelar, B. R., De Oliveira, G. F., Alves, M. S. R., Borges, D. A., Medeiros, M. T., Comendouros, K., \& Scott, F. B. (2017). Eficácia da associação de cipermetrina, clorpirifós, butóxido de piperonila efluazuron contra larvas de Dermatobia hominis em bovinos naturalmente infestados. Brazilian Journal of Veterinary Medicine, 39(1), 28-32. doi: 10.29374/2527-2179.bjvm007817

Fonte de financiamento: Fundação de Apoio à Pesquisa Científica e Tecnológica da Universidade Federal Rural do Rio de Janeiro.

Conflito de interesses: Os autores declaram não haver conflito de interesses que precisam ser informados

Recebido: Março 28, 2017.

Aceito: Setembro 30, 2017

O estudo foi realizado na Universidade Federal Rural do Rio de Janeiro - UFRRJ, Seropédica, RJ, Brasil.

\section{${ }^{*}$ Correspondência}

Fabio Barbour Scott

Programa de Pós-graduação em Ciências

Veterinárias, Instituto de Veterinária,

Universidade Federal Rural do Rio de Janeiro

- UFRRJ

BR 465, Km 7

CEP 23897-035 - Seropédica (RJ), Brasil

E-mail:scott.fabio@gmail.com 


\section{Introdução}

Dermatobia hominis éum dos ectoparasitos mais importantes para a bovinocultura nos países da América Latina. É uma mosca que pertence a Ordem Diptera, Família Oestridae e Subfamília Cuterebrinae. Popularmente é conhecida no Brasil como mosca do berne.Éresponsável por causar míiases furunculosas em bovinos e outros mamíferos, incluindo o homem. Larvas de $D$. hominis causam estresse aos animais, o que resulta em menor ingestão de alimento e, consequentemente, menor ganho de peso, produção de carne e de leito do rebanho. Além disso, sua localização ocorre na região do couro que possui maior valor comercial, o que causa sua depreciação e que acarreta em desvalorização comercial ou o torna impróprio para a industrialização. Acredita-se que o prejuízo econômico ocasiodo por seu parasitismo gire em torno de \$181.55 milhões por ano (Grisi et al., 2002; Moya-Borja, 2003; Grisi et al., 2014).

O controle desta mosca depende unicamente da aplicação de inseticidas nos animais. Apesar de não existirem indícios de resistência, novos ativos ou associações devem testados com a finalidade de facilitar seu controle (Moya-Borja, 2003; Oliveira-Sequeira et al., 2014; Neves et al., 2015).

A cipermetrina é um piretróide sintético, seu mecanismo de ação está no bloqueio dos canais de sódio. Enquanto o clorpirifós é um organofosforado inibidor de alta afinidade da enzima acetilcolinesterase. Ambos são amplamente utilizados no controle de diversos artrópodes parasitos de animais (Taylor, 2001; Oliveira-Sequeira et al., 2014).

O butóxido de piperonila (PBO) tem sido utilizado no controle de pragas agrícolas, pois apresenta sinergismo com piretróides e organofosforados, permitindo assim, a redução da dose e contaminação ambiental ocasionado pelo tratamento destes ectoparasiticidas (Beckel et al., 2006). O fluazuron é um regulador de crescimento de artrópodes inibidor da síntese de quitina que tem sido utilizado com grande frequência em bovinos no controle do carrapato Rhipicephalus microplus (Bull et al., 1996).

O objetivo deste trabalho foi avaliar a eficácia de uma formulação ectoparasiticida contendo a associação de cipermetrina, clorpirifós, butóxido de pireronila e fluazuron na no controle da mosca $D$. hominis em bovinos naturalmente infestados.

\section{Material e métodos}

Este estudo foi aprovado pelo Comitê de Ética de Uso de Animal (CEUA) da Fundação de Apoio à Pesquisa Científica e Tecnológica (FAPUR), da UFRRJ, realizada no dia 11 de junho de 2013.

Para este estudo foram utilizados 20 bovinos, mestiços das raças Gir e Holandês, 12 machos e oito fêmeas, com peso variando de 209 a 432 quilos e identificados por brincos numerados nas duas orelhas. Todos os animais incluídos no estudo apresentaram infestações naturais de larvas de $D$. hominis.

O ranqueamento do estudo foi realizado com base na contagem de larvas vivas em cada animal antes do tratamento. Os dois animais que apresentaram maior contagem de larvas foram sorteados um para cada grupo e o procedimento foi repetido até que estivesse completa as 10 repetições.

No dia do tratamento, os bovinos foram pesados e os animais do grupo tratado foram medicados com a associação de cipermetrina (5mg/kg), clorpirifós $(7 \mathrm{mg} / \mathrm{kg})$, PBO $(5 \mathrm{mg} / \mathrm{kg})$ e fluazuron (2,5mg/kg), na dose de $1 \mathrm{ml}$ para cada 10 quilos de peso vivo, pela via tópica ("pour on") em dose única (Couro Limpo ${ }^{\circledR}$ - Noxon Do Brasil Química e Farmacêutica Ltda). Os animais do grupo controle não receberam nenhum tipo de tratamento.

A avaliação da eficácia foi realizada com sete e com 14 dias após o tratamento, a partir da contagem de larvas vivas presentes nos animais do grupo controle e tratado. O cálculo da eficácia foi realizado com base na fórmula abaixo:

Eficácia (\%) = 100 x (média aritmética de larvas vivas do grupo controle - média aritmética de larvas vivas do grupo tratado) / (média aritmética de larvas vivas do grupo controle).

A análise estatística foi realizada pelo programa estatístico computacional BioStat 5.0 a comparação entre as médias foi realizada pelo teste de Kruskal-Wallis seguido do teste de Student-Newman-Keuls. O nível de significância considerado foi de p $\leq 0,05$ (95\%). 


\section{Resultados}

A média da contagem de larvas vivas antes do tratamento no grupo controle foi de $24,4 \pm 8,11$, enquanto nos animais do grupo tratado foi $24,7 \pm 8,34$, não foi observado diferença estatísticas $(p>0,05)$ entre as médias neste dia experimental.

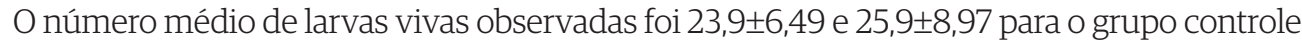

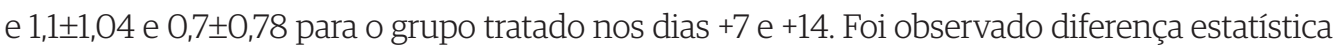
$(\mathrm{p}<0,05)$ entre as médias dos grupos nos dias experimentais após tratamento.

O produto testado apresentou eficácia de 95,26\% para o dia +7 e 97,30\% para o dia +14 . O número de nódulos contendo larvas vivas de $D$. hominis nos grupos controles e tratados sete e 14 dias e após o tratamento e os valores de eficácia estão demonstrados na Tabela 1.

Tabela 1. Contagens individuais de larvas de Dermatobia hominis vivas dentro de nódulos nos bovinos do grupo controle e medicado antes do tratamento e sete e 14 dias após o tratamento da associação de cipermetrina, clorpirifós, butóxido de pireronila e fluazuron empregados por via tópica ("pour on”).

\begin{tabular}{|c|c|c|c|}
\hline \multirow{2}{*}{ Grupo/ animal } & \multicolumn{3}{|c|}{ Número de nódulos contendo larvas vivas de $D$. hominis } \\
\hline & Dia 0 & Dia +7 & Dia +14 \\
\hline \multicolumn{4}{|l|}{ Controle } \\
\hline 15 & 43 & 34 & 37 \\
\hline 17 & 32 & 33 & 37 \\
\hline 18 & 27 & 22 & 21 \\
\hline 21 & 25 & 26 & 29 \\
\hline 12 & 24 & 23 & 25 \\
\hline 16 & 24 & 22 & 37 \\
\hline 30 & 22 & 26 & 29 \\
\hline 14 & 18 & 17 & 15 \\
\hline 27 & 16 & 15 & 12 \\
\hline 23 & 13 & 14 & 17 \\
\hline Média* & $24,4^{\mathrm{a}} \pm 8,11$ & $23,2^{\mathrm{a}} \pm 6,49$ & $25,9^{\mathrm{a}} \pm 8,97$ \\
\hline \multicolumn{4}{|l|}{ Tratado } \\
\hline 25 & 43 & 0 & 0 \\
\hline 19 & 34 & 1 & 1 \\
\hline 29 & 27 & 2 & 1 \\
\hline 20 & 26 & 0 & 2 \\
\hline 26 & 25 & 0 & 0 \\
\hline 11 & 23 & 2 & 0 \\
\hline 13 & 22 & 3 & 1 \\
\hline 24 & 18 & 0 & 2 \\
\hline 22 & 16 & 1 & 0 \\
\hline 28 & 13 & 2 & 0 \\
\hline Média* & $24,7^{\mathrm{a}} \pm 8,34$ & $1,1^{\mathrm{b}} \pm 1,04$ & $0,7^{b} \pm 0,78$ \\
\hline Eficácia \% & - & 95,26 & 97,30 \\
\hline$p$-value & 0,9398 & 0,0005 & 0,0001 \\
\hline
\end{tabular}

*Média Aritmética; Letras diferentes ab significa diferença estatística $(p<0,05)$ entre as médias. 


\section{Discussão}

Também foi possível observar que ao longo do estudo a média de larvas nos animais do grupo controle aumentou, enquanto a do grupo tratado reduziu consideravelmente ao longo dos 14 dias após o tratamento. Este fato pode ser explicado pelo efeito residual do produto no animal, impedindo que novas larvas se desenvolvessem nos animais medicados. Tal efeito também foi observado em outros estudos que utilizaram formulações tópicas contendo associação de organofosforados a piretróides (López-Valencia et al., 2007; Oliveira-Sequeira et al., 2014). O tempo de duração da eficácia residual não foi avaliado neste estudo.

As lactonas macrocíclicas são consideradas os tratamentos mais eficazes no controle das larvas da mosca do berne. No entanto, a associação utilizada neste estudo demonstrou possuir eficácia semelhante a este grupamento químico. Os resultados foram semelhantes ao de outros estudos que utilizaram piretróides e organofosforados (Roncalli \& Usher, 1988; Moya-Borja et al., 1993; Moya-Borja, 2003).

A associação da cipermetrina, clorpirifós, PBO e fluazuron permitiu que doses menores destes ativos fossem utilizados neste estudo mantendo os excelentes níveis de eficácia. Já que, menores doses associado a via de administração "pour on" reduzem a contaminação do ambiente (Beckel et al., 2006; Oliveira-Sequeira et al., 2014).

Uma outra vantagem, seria que associação testada, diferente das lactonas macrocíclicas, apresentam menor impacto sobre fauna coprófila, além de apresenta menor custo de tratamento para o produtor rural (Suarez, 2002; Floate et al., 2005).

Apesar da eficácia de regulador de crescimento de artrópodes do fluazuron não ter sido avaliada neste estudo, vale ressaltar que este possui um longo efeito residual, tendo sua eficácia comprovada para o controle in vivo para o controle de R. microplus (Bull et al., 1996).

\section{Conclusão}

A associação de cipermetrina, clorpirifós, PBO e fluazuron na formulação "pour on" é eficaz no controle de $D$. hominis em bovinos naturalmente infestados.

\section{Referencias}

Beckel, H., Lorini, I., \& Lazzari, S. M. (2006). Efeito do sinergista butóxido de piperonila na resistência de Oryzaephilus surinamensis (L.)(Coleoptera, Silvanidae) a deltametrina e fenitrotiom1. Revista Brasileira de Entomologia, 50(1), 110-114. http://dx.doi.org/10.1590/S0085-56262006000100016.

Bull, M. S., Swindale, S., Overend, D., \& Hess, E. A. (1996). Suppression of Boophilus microplus populations with fluazuron-an acarine growth regulator. Australian Veterinary Journal, 74(6), 468-470. http://dx.doi. org/10.1111/j.1751-0813.1996.tb07575.x. PMid:9006870.

Floate, K. D., Wardhaugh, K. G., Boxall, A. B., \& Sherratt, T. N. (2005). Fecal residues of veterinary parasiticides: nontarget effects in the pasture environment. Annual Review of Entomology, 50(1), 153-179. http://dx.doi. org/10.1146/annurev.ento.50.071803.130341. PMid:15471531.

Grisi, L., Leite, R. C., Martins, J. R., Barros, A. T., Andreotti, R., Cançado, P. H., León, A. A., Pereira, J. B., \& Villela, H. S. (2014). Reassessment of the potential economic impact of cattle parasites in Brazil. Revista Brasileira de Parasitologia Veterinária, 23(2), 150-156. http://dx.doi.org/10.1590/S1984-29612014042. PMid:25054492.

Grisi, L., Massard, C. L., Moya-Borja, G. E., \& Pereira, J. B. (2002). Impacto econômico das principais ectoparasitoses em bovinos no Brasil. A Hora Veterinária, 21, 8-10.

López-Valencia, G., Ruíz-Buitrago, J. D., Avendaño-Rendón, A., \& Ramírez-Posada, J. A. (2007). Evaluación de un producto a base de cipermetrina+ clorpirifos sobre larvas de Dermatobia hominis en bovinos en Titiribí, Antioquia. CES Medicina Veterinaria y Zootecnia, 2, 21-27.

Moya-Borja, G. E. (2003). Erradicação ou manejo integrado das miíases neotropicais das Américas? Pesquisa Veterinária Brasileira, 23(3), 131-138. http://dx.doi.org/10.1590/S0100-736X2003000300006.

Moya-Borja, G. E., Muniz, R. A., Sanavria, A., Goncalves, L. C., \& Rew, R. S. (1993). Therapeutic and persistent efficacy of doramectin against Dermatobia hominis in cattle. Veterinary Parasitology, 49(1), 85-93. http://dx.doi. org/10.1016/0304-4017(93)90227-E. PMid:8236743.

Neves, J. H., Carvalho, N., \& Amarante, A. F. T. (2015). Dermatobia hominis: Potencial risk of resistance to macrocyclic lactones. Veterinary Parasitology, 212(3-4), 483-486. http://dx.doi.org/10.1016/j.vetpar.2015.06.029. PMid:26162561.

Oliveira-Sequeira, T. C. G., Amorim, R. M., Borges, A. S., \& Brant, M. P. R. (2014). Eficácia Terapêutica e Residual de Seis Formulações Inseticidas Sobre o Parasitismo por Larvas de Dermatobia hominis em Bovinos. Veterinária e Zootecnia, 21(1), 177-186. 
Roncalli, R. A., \& Usher, C. B. (1988). Efficacy of ivermectin against Dermatobia hominis in cattle. Veterinary Parasitology, 28(4), 343-346. http://dx.doi.org/10.1016/0304-4017(88)90082-9. PMid:3420795.

Suarez, V. H. (2002). Helminthic control on grazing ruminants and environmental risks in South America. Veterinary Research, 33(5), 563-573. http://dx.doi.org/10.1051/vetres:2002039. PMid:12387490.

Taylor, M. A. (2001). Recent developments in ectoparasiticides. Veterinary journal (London, England : 1997), 161(3), 253-268. http://dx.doi.org/10.1053/tvjl.2000.0549. PMid:11352483. 